\title{
USO DE REDES SOCIALES POR LOS PARLAMENTOS COMO MEDIO DE PARTICIPACIÓN POLÍTICA. ESTUDIO DE CASO LATINOAMERICANO Y EUROPEO
}

\author{
The use of social networks by parliaments as an \\ instrument of political participation. A case study of \\ Europe and Latin America
}

\section{Santiago Giraldo-Luque e Isabel Villegas-Simón}

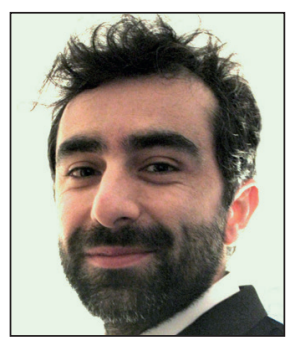

Santiago Giraldo-Luque es profesor del Departamento de Periodismo de la Universitat Autònoma de Barcelona (UAB). Es el coordinador del Master de Investigación en Comunicación y Periodismo de la $U A B$ y el coordinador del Postgrado en Periodismo de Datos de la Universitat de Vic - Universitat Central de Catalunya. Es investigador del Gabinete de Comunicación y Educación de la UAB en donde trabaja temas de participación política; ciudadanía, redes sociales y movimientos sociales, y alfabetización mediática. Es doctor en Comunicación y Periodismo y master en Comunicación y Educación por la $U A B$ y politólogo por la Universidad Nacional de Colombia. http://orcid.org/0000-0003-0024-7081

santiago.giraldo@uab.cat

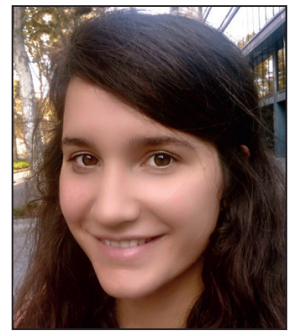

Isabel Villegas-Simón es doctoranda en el Departamento de Comunicación Audiovisual y Publicidad de la Universitat Autònoma de Barcelona (UAB). En 2013, obtuvo el graduado en Comunicación Audiovisual por la Universidad Carlos III de Madrid, y en 2014 finalizó el Master de Investigación en Periodismo y Comunicación en la UAB. Desde 2013 ha participado en proyectos de investigación sobre alfabetización mediática, recepción de contenidos audiovisuales y aspectos culturales de los contenidos de ficción.

http://orcid.org/0000-0003-3064-6876

isamarvillegas@gmail.com

Universitat Autònoma de Barcelona, Departament de Periodismo y Ciencias de la Comunicació Campus de la UAB, Edifici I. 08193 Bellaterra, Barcelona, España

\begin{abstract}
Las redes sociales son consideradas uno de los medios de interacción más importantes de internet, al tiempo que son vistas como espacios de movilización ciudadana, constituyendo canales de información, discusión y participación. Se analiza el uso de las redes sociales por los parlamentos nacionales de diez países como medios para incentivar la participación ciudadana. Atendiendo a los ejes de acción política (Vedel, 2007), el artículo realiza un análisis de contenido longitudinal —con dos años de referencia (2010-2015) - en el que se explora el tipo de redes sociales utilizadas, la evolución de su uso y el alcance que tienen para generar procesos de información, de deliberación y participación. Los resultados muestran que los parlamentos de los países analizados utilizan las redes sociales para comunicar sus actividades y noticias, pero no como espacio deliberativo y participativo incorporado al proceso de toma de decisiones públicas.
\end{abstract}

Palabras clave

Redes sociales; Participación política; Poder legislativo; Ciudadanía activa; Comunicación política; Esfera pública.

\section{Abstract}

Social networks are considered one of the most important developments of the Internet. They are also seen as spaces for social movements in which the channels of information, debate, and participation merge. Using content analysis this article explores social media as an instrument to motivate political participation by citizens from ten countries. Based on the political action axes (Vedel, 2007), a content analysis is conducted to compare the following factors between two years (2010 and 2015): the type of open social networks used, the evolution of their use and potential to generate information, and deliberative and participative processes. The outcomes show that parliaments of the analyzed countries use social media tools to communicate their activities and news. However, they don't use them as a deliberative and participative scenario integrated into the political decision process. 


\section{Keywords}

Social networks; Political participation; e-Parliaments; Active citizenship; Political communication; Public sphere.

Giraldo-Luque, Santiago; Villegas-Simón, Isabel (2017). “Uso de redes sociales por los parlamentos como medio de participación política. Estudio de caso latinoamericano y europeo”. El profesional de la información, v. 26, n. 3, pp. 430437.

https://doi.org/10.3145/epi.2017.may.09

\section{Introducción}

La euforia democrática con la llegada de los medios de comunicación y las tecnologías (Perceval, 2015) ha despertado una ilusión sobre la participación política en la ciudadanía que se ha reflejado en intensas movilizaciones sociales desde la segunda década del siglo XXI (Bennett; Segerberg, 2014; Bimber et al., 2005; Anduiza et al., 2014). Este fenómeno ha sido alimentado por discursos ligados a la transparencia (Lévy, 2002), al final de la corrupción política (Bentivegna, 2006) o al aumento de la participación ciudadana en la toma de decisión (Breindl, 2010). El auge de internet, sin embargo, también ha sido criticado recientemente al evaluar las experiencias de las movilizaciones sociales (Bauman; Bordoni, 2016), los alcances reales de la participación (Giraldo-Luque, 2015), y las dificultades y controles de la participación de multitudes cuyas voces no encuentran recepción en los procesos de decisión política (Zizek, 2010).

Dentro de este debate, a las redes sociales se les ha asignado un papel transcendental. Varios estudios han explorado la influencia de las redes sociales en las movilizaciones ciudadanas (Bond et al., 2012; Kramer et al., 2014; Coviello et al., 2014) y en su uso dentro de acciones colectivas (Anduiza et al., 2014). Sin embargo, el estudio del uso que las instituciones públicas realizan de las redes sociales ha estado ausente de la bibliografía académica. Aun así, en la práctica, una gran parte de las instituciones representativas han apostado por las redes sociales para dar una imagen de transparencia y modernidad democrática. Las redes sociales se presentan como medio para motivar la participación y acercar el ciudadano a la política (Bennett, 2008; Ward et al., 2005; Westling, 2007).

El objetivo del artículo es comparar el uso de las redes sociales por parte de los parlamentos de diez países de América y Europa en dos años (2010-2015). A partir de los ejes de acción política (Vedel, 2007), el estudio realiza una comparación sobre el tipo de redes sociales utilizadas para generar procesos de información, deliberación y toma de decisión política.

\section{Marco teórico}

\subsection{Dos líneas de debate sobre internet y la partici- pación política}

La bibliografía sobre el uso potencial de internet en las instituciones públicas puede ser dividida en dos bandos. En el primero, pueden ubicarse los trabajos que conciben internet y las tecnologías como fuente potencial de empoderamiento y de advenimiento de la sociedad ciberdemocrática
(Lévy, 2002) y de la emancipación sobre el poder estatal y las fuerzas del mercado (Morris; Delafon, 2002). La "Declaración de la independencia del ciberespacio" (Perry, 1996) es uno de los ejemplos más famosos del ciberentusiasmo y el tecnolibertarianismo (Breindl, 2010, p. 1). La Declaración subrayaba que la tecnología constituye una fuente de empoderamiento que desplaza el poder desde las instituciones gubernamentales hacia los ciudadanos individuales. En la misma línea, Lévy (2002) anticipaba el fin de las dictaduras con la llegada de la "ciberdemocracia" y Bentivegna (2006) señalaba el potencial de internet para reconectar a los ciudadanos con los representantes y el renacimiento de la democracia bajo el modelo ateniense.

La "Declaración de la independencia del ciberespacio" (Perry, 1996) subrayaba que la tecnología constituye una fuente de empoderamiento que desplaza el poder desde las instituciones gubernamentales hacia los ciudadanos

Frente a los discursos de esperanza, los frecuentes casos de corrupción y la crisis económica incrementaron la apatía del ciudadano y su alejamiento de la política convencional y sus instituciones. En esas condiciones, los discursos pesimistas sobre la incidencia de internet en el proceso político ganaron apoyo. La segunda perspectiva teórica, la postura crítica, se inicia con las ideas de Van-de-Donk y Tops (1995), quienes denunciaban a internet como un posible instrumento de vigilancia en contra de las libertades. El discurso crítico evoluciona hasta comprobarse que las TIC no han sido suficientes para cambiar el proceso de participación política debido a la reticencia del propio sistema político y a la falta de voluntad de los ciudadanos (Bentivegna, 2006). Los aportes de Margolis y Resnick (2000) destacan a internet como un canal adicional dentro del tradicional juego político, no como un elemento disruptivo. Bauman (Querol, 2016) denuncia a internet, y a las redes sociales en particular, como "zonas de confort" que minan la capacidad de la ciudadanía para interactuar y generar procesos sociales.

Internet ha provocado también cambios en la configuración de la relación entre el ciudadano y los procesos políticos. Bentivegna (2006) señala la pérdida de poder de las instituciones políticas tradicionales, y Cammaerts y Van-Audenhove (2005) observan un ciudadano menos formado por estructuras ideológicas fuertes y procesos políticos formales. El comportamiento ciudadano e internet permiten retomar iniciativas que empoderan a los individuos/colectivos con 
menos recursos transformándolos en actores de las agendas públicas.

A partir del siglo XXI las aplicaciones y las interfaces de colaboración ofertan posibilidades y formas de participación individualizada, basadas en el contenido generado por el usuario. Häyhtiö y Rinne (2008, p. 26) indican que las actividades individuales realizadas en internet para participar políticamente difieren de la participación tradicional en la agenda, los objetivos, la duración temporal y las líneas de actividades. Tal 'evolución' está ligada al proceso general de individualización que da forma a nuevas prácticas políticas, un cambio en el que los ciudadanos tienden a establecer conexiones políticas siguiendo intereses personales y realizar elecciones basadas en estilos de vida más que en ideologías tradicionales.

\subsection{Las redes sociales y la participación política}

Las redes sociales se definen como servicios basados en aplicaciones web que permiten al usuario crear un perfil público o semipúblico dentro de un sistema delimitado de interacción (Boyd; Ellison, 2008). Las redes sociales se organizan y articulan en torno a los usuarios que estructuran, comparten y crean una buena parte del contenido de la plataforma. En su definición, Boyd y Ellison establecen que, aunque la importancia y alcance de las redes sociales están definidos por el perfil y el número de usuarios que las conforman, pueden clasificarse según las posibilidades que el usuario tiene para interactuar con los otros, y de acuerdo con los niveles de privacidad ofertados.

La explosión popular de las redes sociales las ha convertido en el epicentro de diferentes sectores y objetivos. Su creciente impacto despertó la idea de que poseen mecanismos y funciones que permitirían el desarrollo de una nueva esfera pública democrática, colectiva y participativa (Van-Dijck, 2011). Pero esta noción optimista también es cuestionada al explorar la cuestión de cómo los valores públicos, los conceptos legales y los instrumentos económicos se entretejen en su construcción y en su definición. Van-Dijck (2011) parte de la concepción de que las redes sociales son espacios donde diferentes intereses - privados, públicos y corporativos- compiten por dominar las reglas de la interacción social.

Van-Dijck (2011) define las redes sociales como instrumentos comunicativos que formalizan e inscriben un discurso preexistente en la esfera pública. Para la autora son producto de una cultura en la que son las estrategias comerciales las que median en la configuración de las normas de socialización y conectividad, por lo que deben emerger nuevas normas y regulaciones que medien en ellas como nuevos canales de comunicación.

\section{Marco metodológico}

El artículo presenta una investigación exploratoria y longitudinal que utiliza el análisis de contenido para estudiar el uso de las redes sociales en 5 países americanos (Argentina, Chile, Colombia, Ecuador y Estados Unidos) y en 5 europeos (España, Francia, Italia, Suecia y Reino Unido) en dos momentos, 2010 y 2015 . En cada uno de los países fueron analizadas las cámaras que componen el parlamento y las redes sociales que están vinculadas a sus webs oficiales a partir de tres categorías de análisis -información, deliberación y toma de decisión- (Vedel, 2007; Aichholzer; Kuzeluh, 2009; ONU, 2014; Macintosh, 2004). La comparación entre 2010 y 2015 permite observar la evolución en cada una de las categorías. En el anexo se presenta el listado tanto de las cámaras representativas analizadas en cada país de la muestra, así como las redes sociales encontradas y estudiadas.

El análisis de contenido parte de la propuesta de categorías, unidades de contexto y unidades de análisis realizada por Giraldo-Luque (2012) para evaluar el uso que las instituciones públicas hacen de internet en el proceso de participación. Se han seleccionado las unidades de contexto relacionadas con las redes sociales. Cada unidad de contexto refiere a una categoría (informar, deliberar y decidir) y contiene unidades de análisis que identifican los aspectos específicos analizados. La primera unidad de contexto se ubica en el eje de acción de informar y analiza la presencia y el uso comunicativo que realiza la institución para acercarse a la ciudadanía. La segunda unidad de contexto se encuentra en el eje de deliberación y explora el compromiso de la institución en la relación comunicativa que establece con los ciudadanos a través de la red social. Por último, la tercera unidad de análisis se centra en el eje de toma de decisión y examina el compromiso de la institución a incorporar las demandas ciudadanas a los procesos de toma de decisión. La tabla 1 muestra la relación entre categorías, unidades de contexto y unidades de análisis.

Tabla 1. Cuadro de categorías y unidades de análisis

\begin{tabular}{|c|c|c|c|}
\hline Categoría & Informar & Deliberar & Decidir \\
\hline Unidad de contexto & Presencia/Uso informativo & Uso deliberativo & Uso para la toma de decisión \\
\hline \multirow{3}{*}{ Unidades de análisis } & A. Presencia en la red social & $\begin{array}{l}\text { A. Oferta abierta, clara y reglamenta- } \\
\text { da de diálogo }\end{array}$ & $\begin{array}{l}\text { A. Compromiso de la institución } \\
\text { parlamentaria, y de su marco legal, } \\
\text { hacia la oferta de mecanismos de } \\
\text { participación }\end{array}$ \\
\hline & $\begin{array}{l}\text { B. Uso informativo de la red social } \\
\text { (unidireccional). Publicación de } \\
\text { información (agendas, actividad } \\
\text { parlamentaria, noticias) }\end{array}$ & $\begin{array}{l}\text { B. Intencionalidad del ciudadano en } \\
\text { participar expresada en el envío de } \\
\text { comunicaciones }\end{array}$ & $\begin{array}{l}\text { B. Inclusión de los productos de la } \\
\text { participación digital en el proceso } \\
\text { de discusión legislativa y de toma de } \\
\text { decisiones }\end{array}$ \\
\hline & & $\begin{array}{l}\text { C. Obligatoriedad de respuesta } \\
\text { oportuna y precisa por parte de la } \\
\text { institución }\end{array}$ & \\
\hline
\end{tabular}


Tabla 2. Mapa de codificación. Análisis de contenido (sistema de codificación: presencia ponderada)

\begin{tabular}{|c|c|c|c|}
\hline $\begin{array}{l}\text { Categoría - } \\
\text { Unidad de contexto }\end{array}$ & Unidad de análisis & Total & $\begin{array}{l}\text { Valor máximo } \\
\text { de la unidad de } \\
\text { contexto }\end{array}$ \\
\hline \multirow{2}{*}{$\begin{array}{l}\text { Informar - } \\
\text { Presencia y uso informativo }\end{array}$} & Presencia en la red social & $1(x 5)$ & \multirow{2}{*}{10} \\
\hline & Uso informativo de la red social. Publicación de información & $1(x 5)$ & \\
\hline \multirow{3}{*}{$\begin{array}{l}\text { Deliberar - } \\
\text { Uso deliberativo }\end{array}$} & Oferta abierta, clara y reglamentada de diálogo & $1(x 5)$ & \multirow{3}{*}{15} \\
\hline & Intencionalidad del ciudadano en participar expresada en el envío de comunicaciones & $1(x 5)$ & \\
\hline & Obligatoriedad de respuesta por parte de la institución & $1(x 5)$ & \\
\hline \multirow{2}{*}{$\begin{array}{l}\text { Decidir - } \\
\text { Uso para la toma de decisión }\end{array}$} & $\begin{array}{l}\text { Compromiso de la institución parlamentaria, y de su marco legal, hacia la oferta } \\
\text { de mecanismos }\end{array}$ & $1(x 5)$ & \multirow{2}{*}{10} \\
\hline & $\begin{array}{l}\text { Inclusión de los productos de la participación digital en el proceso de discusión } \\
\text { legislativa y de toma de decisiones }\end{array}$ & $1(x 5)$ & \\
\hline
\end{tabular}

El análisis del uso de las redes sociales fue realizado durante los meses de octubre y diciembre, tanto en 2010 como en 2015. En cada red analizada se estudió su comportamiento y el uso que hacía el parlamento - así como la respuesta y la interacción ciudadana- para responder a las unidades de contenido planteadas en cada categoría (tabla 1). El estudio se centró en las publicaciones realizadas por las cámaras en cada red social y, en cada caso, se analizaron entre 40 y 45 publicaciones, así como las características de apertura, interacción, participación y respuesta que las instituciones definían para cada red. Las observaciones realizadas fueron sistematizadas en hojas de codificación por cámara y país (Hernández-Sampieri et al., 2006, p. 367) preparadas en hojas de cálculo sincronizadas (Microsoft Excel) y prediseñadas a partir de la ponderación establecida en las unidades de análisis (tabla 2).

Una gran parte de las instituciones representativas han apostado por las redes sociales para dar una imagen de transparencia y modernidad democrática

El marco cuantitativo se estructura a partir de la relación entre cada una de las unidades de análisis y las redes socia-

Tabla 3. Resultados por unidad de contexto: Presencia e información en redes sociales

\begin{tabular}{|l|c|c|c|c|}
\hline & $\mathbf{2 0 1 0}$ & $\mathbf{2 0 1 5}$ & $\begin{array}{c}\text { Variación en } \\
\text { puntos }\end{array}$ & $\begin{array}{c}\text { Multiplicador } \\
\mathbf{2 0 1 5 / 2 0 1 0}\end{array}$ \\
\hline Promedio & 2,7 & 3,8 & 1,1 & 1,4 \\
\hline Argentina & 0,0 & 2,0 & 2 & $\mathrm{NA}$ \\
\hline Chile & 2,5 & 1,5 & -1 & 0,6 \\
\hline Colombia & 2,5 & 4,5 & 2 & 1,8 \\
\hline Ecuador & 4,0 & 4,0 & 0 & 1,0 \\
\hline Francia & 4,0 & 9,0 & 5 & 2,3 \\
\hline Suecia & 0,0 & 2,0 & 2 & $\mathrm{NA}$ \\
\hline España & 2,0 & 4,0 & 2 & 2,0 \\
\hline Italia & 1,0 & 4,0 & 3 & 4,0 \\
\hline Reino Unido & 8,0 & 6,0 & -2 & 0,8 \\
\hline Estados Unidos & 3,0 & 1,0 & -2 & 0,3 \\
\hline
\end{tabular}

les utilizadas por los parlamentos estudiados - hasta cinco redes sociales por cada objeto analizado-. El análisis sigue la clasificación de presencia ponderada (Bardin, 1986 p. 77)

\section{Los ciudadanos tienden a establecer co-} nexiones políticas siguiendo intereses personales y realizar elecciones basadas en estilos de vida más que en ideologías tradicionales

por la que se mide la presencia o ausencia de los elementos de las unidades de contexto. Para cada unidad de análisis, en la relación parlamento-red social, la puntuación recibida fue de 0 o 1 punto: un punto por la presencia (ningún punto por la ausencia) en cada una de las redes analizadas por cámara de representación. De esta forma, el máximo nivel de puntuación en cada unidad de análisis era de 5 (al analizar un máximo de 5 redes sociales en cada caso). La puntuación final de cada unidad de contexto varía en función del número de unidades de análisis (tabla 2).

\section{Resultados}

\subsection{Presencia e información en redes sociales}

La primera unidad de contexto presenta un leve aumento en los resultados comparados entre 2010 y 2015 . Las dos unidades de análisis tienen un valor promedio más alto, aunque ese aumento signifique tan solo 1,1 puntos de crecimiento positivo para la unidad de contexto analizada (tabla 3 ).

Los datos permiten identificar tres países que disminuyen sus puntuaciones. El caso más significativo es los Estados Unidos. Es el país que más redujo la puntuación y sus resultados en 2015 lo ubican a la cola de la muestra. Chile y Reino Unido también reducen sus resultados, aunque el país europeo mantiene una posición destacada en el escalafón, sólo superada por Francia, el país que más creció en sus resultados durante el período analizado. La caída de los países señalados es evidenciada en ambas unidades de contexto (tabla 4), una correspondencia lógica como consecuencia de la reducción de su presencia en las redes sociales.

A la inversa, Italia y Francia han sido los países que más han aumentado su puntuación. Italia ha mejorado especialmente 
Tabla 4. Resultados por unidad de análisis. Unidad de contexto 1. Presencia e información en redes sociales

\begin{tabular}{|l|l|l|l|l|}
\hline \multirow{2}{*}{ Unidad de análisis } & \multicolumn{2}{|c|}{$\begin{array}{c}\text { Presencia en redes } \\
\text { sociales }\end{array}$} & \multicolumn{2}{c|}{$\begin{array}{c}\text { Uso informativo del } \\
\text { perfil }\end{array}$} \\
\cline { 2 - 5 } & $\mathbf{2 0 1 0}$ & $\mathbf{2 0 1 5}$ & $\mathbf{2 0 1 0}$ & $\mathbf{2 0 1 5}$ \\
\hline Promedio & 1,55 & 1,95 & 1,15 & 1,85 \\
\hline Argentina & 0 & 1 & 0 & 1 \\
\hline Chile & 1,5 & 1 & 1 & 0,5 \\
\hline Colombia & 1,5 & 2,5 & 1 & 2 \\
\hline Ecuador & 2 & 2 & 2 & 2 \\
\hline Francia & 2,5 & 4,5 & 1,5 & 4,5 \\
\hline Suecia & 0 & 1 & 0 & 1 \\
\hline España & 1 & 2 & 1 & 2 \\
\hline Italia & 1 & 2 & 0 & 2 \\
\hline Reino Unido & 4 & 3 & 4 & 3 \\
\hline Estados Unidos & 2 & 0,5 & 1 & 0,5 \\
\hline
\end{tabular}

en el uso informativo que realiza de las redes. Francia mejoró sus resultados sobre todo en la segunda unidad de análisis. El caso francés llama la atención al ser el único país cercano a obtener los 10 puntos máximos de la unidad de contexto (tabla 4). Cabe destacar que Argentina, Suecia, España e Italia, con puntuaciones nulas o muy bajas en 2010 , han realizado esfuerzos por incorporar su presencia en redes sociales y por realizar alguna función informativa en ellas.

Estados Unidos es el país que más redujo la puntuación y sus resultados en 2015 lo ubican a la cola de la muestra. En cambio, Francia fue el país que más creció y el que está mejor situado

En términos de unidades de análisis de contenido (tabla 4), destaca el uso informativo del perfil en la red social frente a la presencia de la institución en los medios sociales. La diferencia en el aumento indica una pequeña mayor implicación de los parlamentos estudiados por incrementar el uso informativo de las redes sociales.

\subsection{Uso deliberativo de las redes sociales}

El análisis señala que hay un descenso mínimo en el eje deliberativo del estudio. La tabla 5 da cuenta de un intenso movimiento en el comportamiento de los parlamentos, guiado por la apertura de canales de comunicación en las redes sociales, pero también, por el cierre de otros.

Mientras tan sólo tres países mantienen los niveles de 2010 (Colombia, Suecia y Estados Unidos), hay cuatro países que retroceden frente al 2010 (Chile, Ecuador, Francia y Reino Unido). Vale la pena destacar el caso de Suecia y los Estados Unidos quienes no han apostado por el uso de redes sociales para promover la deliberación en ninguno de los momentos evaluados. El caso colombiano responde tanto al incremento del uso de Facebook en una de las cámaras como al uso unidireccional del Twitter en los últimos años,
Tabla 5. Resultados por unidad de contexto: uso deliberativo

\begin{tabular}{|l|c|c|c|c|}
\hline & $\mathbf{2 0 1 0}$ & $\mathbf{2 0 1 5}$ & $\begin{array}{c}\text { Variación } \\
\text { en puntos }\end{array}$ & $\begin{array}{c}\text { Multiplicador } \\
\mathbf{2 0 1 5 / 2 0 1 0}\end{array}$ \\
\hline Promedio & 1,9 & 1,7 & $-0,2$ & 0,9 \\
\hline Argentina & 0,0 & 2,0 & 2,0 & NA \\
\hline Chile & 2,0 & 0,0 & $-2,0$ & NA \\
\hline Colombia & 2,0 & 2,0 & 0,0 & 1,0 \\
\hline Ecuador & 4,0 & 2,0 & $-2,0$ & 0,5 \\
\hline Francia & 5,0 & 4,0 & $-1,0$ & 0,8 \\
\hline Suecia & 0,0 & 0,0 & 0,0 & NA \\
\hline España & 1,0 & 2,0 & 1,0 & 2,0 \\
\hline Italia & 0,0 & 2,0 & 2,0 & NA \\
\hline Reino Unido & 5,0 & 3,0 & $-2,0$ & 0,6 \\
\hline Estados Unidos & 0,0 & 0,0 & 0,0 & NA \\
\hline
\end{tabular}

un hecho que ha convertido la red social de microblogging en una herramienta más informativa que deliberativa.

Los retrocesos encontrados siguen la misma dinámica que en Colombia: la mayoría de países analizados utilizan Twitter únicamente para enviar mensajes, pero no para garantizar o promover un proceso de deliberación a través de los 140 caracteres. El proceso deliberativo encuentra en Facebook un ingrediente de promoción a través de las páginas de los cuerpos representativos que tienen habilitados los espacios de comentarios para los ciudadanos (Ecuador, Francia y Reino Unido). El fenómeno chileno es aún más sorprendente porque las cámaras han optado por Twitter como única red social de comunicación con los ciudadanos.

\section{La mayoría de países analizados utilizan Twitter únicamente para enviar mensa- jes, pero no para garantizar o promover un proceso de deliberación}

Argentina, España e Italia deben el aumento de su puntuación al cambio de estrategias en el uso de redes sociales. En 2015 los países indicados contaban con un canal de tipo más deliberativo en la red social Facebook, lo que permitía al ciudadano realizar mensajes directos a la institución, así como interactuar - sin obligatoriedad de respuesta, como se aprecia en la tercera unidad de análisis estudiada (tabla $6)$ - con los mensajes y las informaciones dadas por el parlamento.

Los resultados detallados por unidad de análisis (tabla 6) demuestran una reducción en los promedios generales en dos de las tres unidades. En cualquier caso, indican una amplia distancia frente a los máximos niveles posibles de cada unidad (5 puntos). Al mismo tiempo, denotan escasos niveles de mejora en los países y una falta de compromiso de parte de las instituciones frente a las discusiones o al impacto de las comunicaciones publicadas en sus redes sociales. 


\subsection{Uso de las redes sociales para la toma de decisiones}

Los resultados poco significativos en la tercera unidad de contexto obligan a simplificar la presentación de los resultados (tabla 7). Los únicos países que obtienen puntuación en la unidad son Francia y Reino Unido, dos países que incluyen algunas regulaciones y compromisos de utilización de los comentarios y propuestas de sus ciudadanos, realizados en los espacios de participación reglados en sus mismas páginas web y algunos otros espacios de interacción ciudadana.

Los resultados no muestran diferencias entre un año y otro $y$, aunque se debe reconocer el compromiso de Francia y Reino Unido, sus alcances son muy limitados. Ninguno de los otros países estudiados realiza esfuerzos por asumir compromisos o por darle mayor visibilidad a los mecanismos de participación democrática.

Los resultados indican un ligero aumento en el uso de las redes sociales por parte de los parlamentos en los últimos cinco años, que disminuye conforme se avanza en los ejes de acción política que implican un mayor compromiso institucional

\section{Discusión}

Los resultados indican un ligero aumento en el uso de las redes sociales por parte de los parlamentos en los últimos cinco años, que disminuye conforme se avanza en los ejes de acción política (Vedel, 2007) que implican, progresivamente, un mayor compromiso institucional. Es posible cuestionar los discursos que conciben las redes sociales como escenarios potenciales para favorecer la participación política (Bentivegna, 2006) y de reconexión con el ciudadano desencanta-

Tabla 7. Resultados por unidad de contexto: uso para la toma de decisión

\begin{tabular}{|l|c|c|c|c|}
\cline { 2 - 5 } \multicolumn{1}{c|}{} & $\mathbf{2 0 1 0}$ & $\mathbf{2 0 1 5}$ & $\begin{array}{c}\text { Variación en } \\
\text { puntos }\end{array}$ & $\begin{array}{c}\text { Multiplicador } \\
\mathbf{2 0 1 5 / 2 0 1 0}\end{array}$ \\
\hline Promedio & 0,2 & 0,2 & 0 & 0,0 \\
\hline Francia & 1,0 & 1,0 & 0 & 0,0 \\
\hline Reino Unido & 1,0 & 1,0 & 0 & 0,0 \\
\hline
\end{tabular}

Tabla 8. Resultados por unidad de análisis. Unidad de contexto 3. Uso de las redes sociales para la toma de decisión

\begin{tabular}{|l|c|c|c|c|}
\hline \multirow{2}{*}{$\begin{array}{c}\text { Unidad de } \\
\text { análisis }\end{array}$} & \multicolumn{2}{c|}{$\begin{array}{c}\text { Compromiso de oferta } \\
\text { de herramientas de } \\
\text { participación }\end{array}$} & \multicolumn{2}{|c|}{$\begin{array}{c}\text { Inclusión de los } \\
\text { resultados de la } \\
\text { participación }\end{array}$} \\
\cline { 2 - 5 } & $\mathbf{2 0 1 0}$ & $\mathbf{2 0 1 5}$ & $\mathbf{2 0 1 0}$ & $\mathbf{2 0 1 5}$ \\
\hline Promedio & 0,1 & 0,1 & 0,1 & 0,1 \\
\hline Francia & 0,5 & 0,5 & 0,5 & 0,5 \\
\hline Reino Unido & 0,5 & 0,5 & 0,5 & 0,5 \\
\hline
\end{tabular}

do con los procesos informativos, deliberativos y de toma de decisión (Westling, 2007). Por el contrario, los datos que se han obtenido demuestran un uso precario y tradicionalista de las redes sociales (Margolis; Resnick, 2000), una visión de internet como una canal adicional y no como un elemento disruptivo en la disputa política. Los datos evidencian que el principal uso que las instituciones representativas analizadas dan a las redes sociales es presencial e informativo. Vale decir, además, que cada vez son más limitadas las posibilidades de las mismas redes para garantizar espacios de debate, deliberación y participación real de la ciudadanía dentro de un proceso de construcción de decisiones.

Frente a los que pronosticaban que las redes sociales serían elementos disruptivos y creativos en la reconciliación y re-conexión con la ciudadanía, han triunfado los más pesimistas

Frente a la expansión y masificación del uso de las redes sociales (Boyd; Ellison, 2008) los parlamentos se mantienen inmóviles y, hasta ahora, no han sido capaces de canalizar y dar respuesta a la intensidad de movimientos sociales que sí han penetrado nuevos espacios comunicativos (Bennett; Segerberg, 2014; Bimber et al., 2005; Anduiza et al., 2014). Las ideas que se desprenden de los resultados realzan las dificultades y controles a los que se enfrentan las instituciones para articular y recoger la multitud de voces que se encuentran en los espacios cibernéticos (Zizek, 2010). El sistema político sigue siendo reticente a asumir las tecnologías de la información y la comunicación como herramientas de re-conexión con los ciudadanos (Bentivegna, 2006).

El contraste entre la puntuación de cada uno de los ejes analizados entronca con las propuestas de Van-Dijck (2011) quien, al considerar las redes sociales como un espacio de batalla entre los actores públicos, privados y corporativos en el que se disputa la construcción de la normativa social, evidencia el escaso papel que las instituciones representati- 
vas juegan en dicho enfrentamiento. A la inversa las instituciones de la muestra utilizan las redes sociales para expandir el mismo discurso ya publicado en el resto de sus canales de comunicación. En lugar de aprovechar los mecanismos y recursos de las redes sociales, identificadas como herramientas potencialmente decisivas en la construcción de la una nueva esfera pública de deliberación y participación, las instituciones representativas las emplean como meros canales de réplica informativa o como placebo democrático ante una ciudadanía anestesiada (Giraldo-Luque, 2015).

\section{Conclusiones}

Los parlamentos analizados no utilizan todavía las redes sociales como herramientas para promover la deliberación y la participación política de la ciudadanía. El empleo de las redes sociales es prácticamente testimonial, destinándolas a la transmisión de información, que también es reproducida en sus otros canales de comunicación. Frente a los discursos y voces que pronosticaban a las redes sociales como elementos disruptivos y creativos en la reconciliación y re-conexión con la ciudadanía, el rol desempeñado por los parlamentos ha dado el triunfo a las voces más pesimistas. Los espacios de participación siguen cerrados a canales y procedimientos tradicionales.

La crisis de legitimidad y confianza que sufre el sistema representativo puede ampliarse si los parlamentos no desempeñan un rol más activo en la configuración y el uso del espacio que abren las redes sociales

Al margen de esta actitud, las redes sociales sí se han impregnado de una intensa actividad política encauzada por movimientos y fuerzas políticas que se sitúan fuera de las formas institucionales. La canalización de la participación política en redes sociales por organismos, movimientos o plataformas ciudadanas, puede abrir aún más la brecha entre la ciudadanía y las instituciones públicas. La crisis de legitimidad y confianza que sufre el sistema representativo puede ampliarse si los parlamentos no desempeñan un rol más activo en la configuración y el uso del espacio que abren las redes sociales. La exploración de mecanismos que favorezcan la apertura de un nuevo espacio público en redes sociales, la voluntad política que se esconde tras las decisiones que determinan la actividad de las instituciones, la falta de interés sobre una figura política desprestigiada como lo es el parlamento y la predisposición de la ciudadanía para participar en la deliberación y la toma de decisión a través de nuevos mecanismos, son algunos de los escenarios abiertos como posibles puntos explicativos de las ausencias/carencias halladas en el estudio realizado.

\section{Referencias}

Aichholzer, Georg; Kuzeluh, Ulrike (2009). Dimensions and approaches for the evaluation of e-democracy. Strasbourg: Document Cahde (The Council of Europe's ad hoc Committee on E-Democracy), CAHDE (2009) 5E.

\section{https://goo.gl/Kpy5yRf}

Anduiza, Eva; Cristancho, Camilo; Sabucedo, José-Luis (2014). "Mobilization through online social networks: the political protest of the indignados in Spain". Information, communication, society, v. 17, n. 6, pp. 750-764. https://doi.org/10.1080/1369118x.2013.808360

Bardin, Laurence (1986). El análisis de contenido. Madrid: Akal. ISBN: 8476000936

Bauman, Zygmun; Bordoni, Carlo (2016). Estado de crisis. Barcelona: Paidós. ISBN: 9788449331824

Bennett, W. Lance (2008). Civic life online: Learning how digital media can engage youth. Cambridge: The MIT Press. ISBN: 9780262524827

Bennett, W. Lance; Segerberg, Alexandra (2014). The logic of connective action. Digital Media and the Personalization of Contentious Politics. Cambridge University Press. https://doi.org/10.1017/cbo9781139198752.002

Bentivegna, Sara (2006). "Rethinking politics in the worlds of ICT". European journal of communication, v. 21, n. 3, pp. 331-343. http://ejc.sagepub.com/cgi/content/abstract/21/3/331

Bimber, Bruce; Flanagin, Andrew; Stohl, Cynthia (2005). "Reconceptualizing collective action in the contemporary media environment". Communication theory, v. 15, n. 4, pp. 389-413. https://goo.gl/SKRCLa

Bond, Robert M.; Fariss, Christopher J.; Jones, Jason J.; Kramer, Adam D. I.; Marlow, Cameron; Settle, James F.; Fowler, James H. (2012). "A 61-million-person experiment in social influence and political mobilization". Nature, v. 489, pp. 295-298. https://doi.org/10.1038/nature11421

Boyd, Danah M.; Ellison, Nicole B. (2008). "Social network sites: Definition, history and scholarship". Journal of computer-mediated communication, v. 13, n. 1, pp. 210-203. https://doi.org/10.1111/j.1083-6101.2007.00393.x

Breindl, Yana (2010). "Critique of the democratic potentialities of the internet: A review of current theory and practice". Triple C: Cognition, communication, co-operation, v. 8, n. 1, pp. 43-59. ISBN: $1726670 \mathrm{X}$

Cammaerts, Bart; Van-Audenhove, Leo (2005). “Online political debate, unbounded citizenship and the problematic nature of a transnational public sphere". Political communication, v. 22, n. 2, pp. 179-196. https://doi.org/10.1080/10584600590933188

Coviello, Lorenzo; Sohn, Yunkyu; Kramer, Adam D. I.; Marlow, Cameron; Franceschetti, Massimo; Christakis, Nicholas A.; Fowler, James H. (2014). "Detecting emotional contagion in massive social networks". PLoS one, v. 9, n. 3, e90315. https://doi.org/10.1371/journal.pone.0090315

Giraldo-Luque, Santiago (2015). Més enllà de Twitter. De l'expressió indignada a l'acció política. Vic: Eumo Editorial. ISBN: 9788497665537

Giraldo-Luque, Santiago (2012). “Modelo de análisis del uso de internet para promover la participación ciudadana en los procesos de toma de decisión pública (poderes ejecutivo y legislativo)". Signo y pensamiento, v. 31, n. 61, pp. 18-36. 
https://goo.gl/yLqQvA

Häyhtiö, Tapio; Rinne, Jarmo (2008). “Introduction: Seeking the citizenry on the internet - Emerging virtual creativity". En: Häyhtiö, T., Rinne, J. (eds.). Net working/networking: Citizen initiated internet politics. Tampere: Tampere University Press, pp. 11-38. ISBN: 9789514474644

Hernández-Sampieri, Roberto; Fernández-Collado, Carlos; Baptista-Lucio, María-del-Pilar (2008). Metodología de investigación. México D. F.: McGraw-Hill Interamericana. ISBN: 9701057532

Kramer, Adam D. I.; Guillory, Jamie E.; Hancock, Jeffrey T. (2014). "Experimental evidence of massive-scale emotional contagion through social networks". Procs of the National Academy of Sciences, v. 111, n. 24, pp. 8788-8790.

https://doi.org/10.1073/pnas.1320040111

Lévy, Pierre (2002). Ciberdemocracia. Ensayo sobre filosofía política. Barcelona: Editorial UOC. ISBN: 8497881338

Macintosh, Ann (2004). "Characterizing e-participation in policy-making". En: Procs of the $37^{\text {th }}$ Hawaii intl conf on system sciences, pp. 5-8.

https://doi.org/10.1109/hicss.2004.1265300

Margolis, Michael; Resnick, David (2000). Politics as usual: The cyberspace "revolution". Londres: Sage. ISBN: 978 0761913306

Morris, Dick; Delafon, Gilles (2002). Vote.com ou comment internet va révolutionner la politique. Paris: Plon. ISBN: 2 259194729

ONU (2014). UN e-Government Suvey 2014. E-government for the future we want. New York: United Nations. https://goo.gl/8NJTWR

Perceval, José-María (2015). Historia mundial de la comunicación. Madrid: España. ISBN: 9788437634227

Perry, John (1996). Declaración de independencia del ciberespacio. Suiza.

http://www.uhu.es/ramon.correa/nn_tt_edusocial/documentos/ docs/declaracion_independencia.pdf

Querol, Ricardo (2016). "Zygmunt Bauman: 'Las redes sociales son una trampa'”. El país, 9 de enero de 2016. https://goo.gl/g48rLk

Van-de-Donk, Wim; Tops, Pieters (eds). (1995). Orwell in Athens. A perspective on informatization and democracy. New York: IOS Press. ISBN: 9789051992199

Van-Dijck, José (2011). "Facebook as a tool for producing sociality and connectivity". Television \& new media, v. 13, n. 2, pp. 1-17. https://doi.org/10.1177/1527476411415291

Vedel, Thierry (2007). Les usages politiques d'internet, La démocratie électronique. Paris: La Documentation française.

Ward, Stephen; Gibson, Rachel; Lusoli, Wainer (2005). “Old politics, new media: Parliament, the public and the internet", En: Political Studies Association conference, University of Leeds. https://goo.gl/1vwCVH

Westling, Mike (2007). "Expanding the public sphere: The impact of Facebook on political communication". UW Madison. https://goo.gl/2R7Nuv
Zizek, Slavoj (2010). El acoso de las fantasías. Madrid: Siglo XXI. ISBN: 9788432314001

Anexo

Redes sociales analizadas por cada país de la muestra en 2010 y $2015 *$

\begin{tabular}{|c|c|c|c|}
\hline País & $\begin{array}{c}\text { Cámaras } \\
\text { representativas }\end{array}$ & $\begin{array}{c}\text { Redes sociales en } \\
2010 \\
\end{array}$ & $\begin{array}{c}\text { Redes sociales en } \\
2015 \\
\end{array}$ \\
\hline \multirow{3}{*}{$\begin{array}{l}\text { Argen- } \\
\text { tina }\end{array}$} & \multirow{2}{*}{$\begin{array}{l}\text { Senado de la } \\
\text { Nación }\end{array}$} & \multirow{2}{*}{$\begin{array}{l}\text { No se encontraron } \\
\text { redes sociales }\end{array}$} & Facebook \\
\hline & & & Twitter \\
\hline & $\begin{array}{l}\text { Cámara de } \\
\text { Diputados de la } \\
\text { Nación }\end{array}$ & $\begin{array}{l}\text { No se encontraron } \\
\text { redes sociales }\end{array}$ & $\begin{array}{l}\text { No sé encontraron } \\
\text { redes sociales }\end{array}$ \\
\hline \multirow{3}{*}{ Chile } & Senado & Twitter & Twitter \\
\hline & \multirow{2}{*}{$\begin{array}{l}\text { Honorable } \\
\text { Cámara de los } \\
\text { Diputados }\end{array}$} & Facebook & \multirow[b]{2}{*}{ Twitter } \\
\hline & & Twitter & \\
\hline \multirow{5}{*}{$\begin{array}{l}\text { Colom- } \\
\text { bia }\end{array}$} & \multirow{3}{*}{$\begin{array}{l}\text { Senado de la } \\
\text { República de } \\
\text { Colombia }\end{array}$} & Facebook & Facebook \\
\hline & & Twitter & Twitter \\
\hline & & Linkedln & Linkedln \\
\hline & \multirow{2}{*}{$\begin{array}{l}\text { Cámara de Repre- } \\
\text { sentantes }\end{array}$} & \multirow{2}{*}{$\begin{array}{l}\text { No se encontraron } \\
\text { redes sociales }\end{array}$} & Facebook \\
\hline & & & Twitter \\
\hline \multirow{2}{*}{ Ecuador } & \multirow{2}{*}{$\begin{array}{l}\text { Asamblea Nacio- } \\
\text { nal de Ecuador }\end{array}$} & Facebook & Facebook \\
\hline & & Twitter & Twitter \\
\hline \multirow{9}{*}{ Francia } & \multirow{5}{*}{ Sénat } & Facebook & Facebook \\
\hline & & Twitter & Twitter \\
\hline & & Linkedln & Dailymotion \\
\hline & & \multirow{2}{*}{ Flickr } & Google+ \\
\hline & & & Instagram \\
\hline & \multirow{4}{*}{$\begin{array}{l}\text { Assemblée } \\
\text { Nationale }\end{array}$} & \multirow{4}{*}{ LinkedIn } & Facebook \\
\hline & & & Twitter \\
\hline & & & Dailymotion \\
\hline & & & Google+ \\
\hline Suecia & Riksdag & $\begin{array}{l}\text { No se encontraron } \\
\text { redes sociales }\end{array}$ & Twitter \\
\hline \multirow[t]{2}{*}{ España } & Senado & $\begin{array}{l}\text { No se encontraron } \\
\text { redes sociales }\end{array}$ & Twitter \\
\hline & Congreso & Twitter & Twitter \\
\hline \multirow{4}{*}{ Italia } & \multirow{2}{*}{$\begin{array}{l}\text { Senato della } \\
\text { Repubblica }\end{array}$} & \multirow{2}{*}{ Linkedln } & Twitter \\
\hline & & & YouTube \\
\hline & \multirow{2}{*}{$\begin{array}{l}\text { Camera dei } \\
\text { Deputati }\end{array}$} & \multirow{2}{*}{ Linkedln } & Facebook \\
\hline & & & YouTube \\
\hline \multirow{9}{*}{$\begin{array}{l}\text { Reino } \\
\text { Unido }\end{array}$} & \multirow{5}{*}{ House of Lords } & Facebook & Facebook \\
\hline & & Twitter & Twitter \\
\hline & & Friendfeed & YouTube \\
\hline & & \multirow{2}{*}{ Flickr } & Flickr \\
\hline & & & Google + \\
\hline & & Facebook & \\
\hline & House of & Twitter & Twitter \\
\hline & Commons & Friendfeed & Iwitcer \\
\hline & & Flickr & \\
\hline & U.S. Senate & Facebook & Twitter \\
\hline Estados & U.s. senate & Linkedln & IWItter \\
\hline Unidos & House of & Facebook & No se encontraron \\
\hline & representatives & Linkedln & redes sociales \\
\hline
\end{tabular}

* Las redes sociales analizadas en cada año del estudio corresponden a las que estaban vinculadas a la web de cada uno de los parlamentos (cámaras) estudiados. 